Bangladesh J. Bot. 49(4): 1127-1134, 2020 (December)

\title{
SILVER NANOPARTICLES FROM ZIZIPHUS SPINA-CHRISTI (L.) DESF. FRUITS EXTRACT AND THEIR ANTIMICROBIAL ACTIVITY
}

\author{
Mahmoud Mostafa ${ }^{1 *}$, Ahmed Al-Emam ${ }^{2,3}$, Mahmoud Sayed ${ }^{4,5}$, \\ Saad Aiamri ${ }^{6}$, Huda Alghamdil, Ali Shati, Sulaiman Alrumman \\ and Abdul Khaleg Alfaify \\ Department of Biology, College of Science, King Khalid University, Abha-9004, \\ Kingdom of Saudi Arabia
}

Keywords: Zizphus spina-christi, Fruits, Antimicrobial activity, Silver nanoparticles

\begin{abstract}
An easy and ecofriendly method to synthesize silver nanoparticles from different stages of ripening fruit extracts of Ziziphus spina-christi is demonstrated. UV-Vis spectroscopy showed a surface resonance peak at 423, 428 and $438 \mathrm{~nm}$ corresponding to the formation of AgNPs from ripened, unripened and young fruits, respectively. On the basis of XRD analysis the particle size of three stages of fruit ripening was found to range between 16.5 and $50.76 \mathrm{~nm}$ and all had silver metal which was confirmed by EDX. The inhibitory efficacy of AgNPs increased with the ripening progress such as ripened fruit > unripened fruit > young fruit against all tested human pathogenic microbes.
\end{abstract}

\section{Introduction}

Ziziphus spina-christi (L.) Desf. is an evergreen tree native to most countries in Africa and southern and Western Asia. It grows a height of $20 \mathrm{~m}$ and the trunk of diameter of $60 \mathrm{~cm}$. There are approximately 50 species distributed across the tropical regions of Asia, Africa, and America and in the temperate regions of both hemispheres (Townsend and Guest 1974). Z. spina-christi is considered to be one of the few truly native tree species of Saudi Arabia that is still growing, along with many newly introduced invasive weed species (Mandaville and Mandaville 1990, Saied et al. 2008). The plant may be found in severe drought conditions as it has been adapted to various environmental patterns in the Kingdom of Saudi Arabia because it has remarkable genetic variation (Moustafa et al. 2016). In folk medicine, all parts of $Z$. spina-christi are used by local Arabs to promote a safe lifestyle (Saied et al. 2008). The plant extract has been documented as a health-promoting compound, with anticathartic, astringent, diuretic, and tonic properties (Duke and Ayensu 1985). Water extract of Z. spina-christi found to be useful in the treatment of hepatic fibrosis (Amin and Mahmoud-Ghoneim 2009). The plant was mentioned in ancient papyri for healing of wounds and anti-swellings (Kadioglu et al. 2016). In Sudan and in Egypt, fruits are usually eaten for the treatment of diarrhoea, as antispasmodic and for malaria (El-Kamali and ElKhalifa 1999).

It is therefore of significant concern to search the inhibitory effect of AgNPs from Z. spinachristi that can be applied in the field of nanotechnology as a cost-effective and eco-friendly. This study was undertaken to synthesize and characterise changes in silver nanoparticles gained from fresh fruits extract of $Z$. spina-christi associated with successive stages of ripening and evaluate their antimicrobial efficacy against human pathogens.

*Author for correspondence: <mfmostfa@kku.edu.sa>. ${ }^{1}$ Department of Botany and Microbiology, Faculty of Science, South Valley University, Qena, Egypt. ${ }^{2}$ Department of Pathology, College of Medicine, King Khalid University, Abha, Kingdom of Saudi Arabia. ${ }^{3}$ Department of Forensic Medicine and Clinical Toxicology, Faculty of Medicine, Mansoura, University, Mansoura, Egypt. ${ }^{4}$ Department of Physics, Faculty of Science, King Khalid University, P. O. Box 9004, Abha, Saudi Arabia. ${ }^{5}$ Department of Physics, Faculty of Science, Al-Azhar University, P. O. 71452, Assiut, Egypt. ${ }^{6}$ Prince Sultan Bin Abdulaziz Center For Environmental and Tourism Research and Studies, King Khalid University, Kingdom of Saudi Arabia. 


\section{Materials and Methods}

Z. spina-christi fruits were collected from Asir region, KSA during the growing season of 2018-2019 and the experimental work was carried out at the Biology Department, Faculty of Science, King Khalid University, KSA. Fruits were sampled from growing sites at 3 different intervals during successive fruit ripening stages (young, unripined and ripened fruit).

At each time $3 \mathrm{~g}$ of peal of fruits was separated from the seeds, washed thoroughly with distilled water and crushed into fine particles with the addition $3 \mathrm{ml}$ of DW using mortar and pestle. Each fruit peal from various stages was filtered carefully using Whatman filter paper (Sigma-Aldrich).

For formulation of silver nanoparticles (AgNPs), silver nitrate (E. Merck, Germany), was used. One $\mathrm{ml}$ from each extract was added drop wise to $10 \mathrm{ml}(0.5 \mathrm{mM})$ aqueous $\mathrm{AgNO}_{3}$ solution in glass test tubes and heated in water bath at $70^{\circ} \mathrm{C}$. Once after the addition of the extract within $30 \mathrm{sec}$ a distinct color change was observed as the solution changed into dark yellow and then transformed into dark brown.

To evaluate the differences between the peak formations of the synthesized silver nanoparticles from three types of extract, the absorption spectrum was taken at $300-540 \mathrm{~nm}$ with a UV-vis-spectrophotometer (HITACHI, Model U-2800 spectrophotometer). De-ionized water was used instead of extract sample extract as a blank.

Crystallinity was demonstrated at room temperature by the X-ray diffraction technique (XRD) using the Shimadzu LabX-XRD-6000 diffractometer with CuK5-007 $\left(\lambda=1.54056 \mathrm{~A}^{\circ}\right)$ radiation and secondary monochromator linked to the Shimadzu program with the pdf-2 library for XRD data analysis. The nanocrystalline size was determined using the equation of Debye-Scherrer formula: $(\mathrm{D}=\mathrm{k} \lambda / \beta \cos \theta$, (D, particle diameter size); $\mathrm{k}$, a constant equal1; $\lambda$, (wavelength of $\mathrm{X}$-ray source $(0.1541 \mathrm{~nm})) ; \beta$, (the full width at half maximum (FWHM)) and $\theta$ (diffraction angle matching to the lattice plane (111)).

The size of particles and existence of metallic silver in the synthesized AgNPs were further verified by Energy (SEM; Hitachi S4800) equipped with an energy-dispersive X-ray spectroscope (Bianco et al. 2016).

Microbial strains, namely Proteus mirabilis, Psedomonas aeruginosa, Staphylococcus aureus, Shigella flexneri, Klebsiella oxytoca, Micrococcus luteus and Candida albicans were received from the Microbiology Laboratory, Faculty of Medicine, King Khalid University, KSA. Bacterial strains were sub-cultured in sterilized nutrient broth at $37^{\circ} \mathrm{C}$ for one day for further use.

The efficacy of antimicrobial activities of synthesised nanoparticles of fruits peal of $Z$. spinachristi was accomplished by using well diffusion methods (Moustafa and Alrumman 2015). The $20 \mathrm{ml}$ from previously sterilised Mueller Hinton agar (Oxoid, England) medium was poured into disposable sterile Petri dish. From each microbial strain $0.1 \mathrm{ml}$ of standardised inoculum was spread carefully onto the agar surface by using a sterile L-shaped glass. Six millimetre diameter well were made by using a sterile cork-borer. Each well was filled by $0.1 \mathrm{ml}$ of the synthesised $\mathrm{AgNPs}$ of pealed fruits. Then all plates were kept at $22^{\circ} \mathrm{C}$ for approximately one hour for extracts diffusion into the agar. Each extract was assayed in triplicate and sterile dimethyl sulfoxide (DMSO), was served as a negative control, and cefoxitin $(30 \mathrm{mcg})$ as a positive control. Each plate was incubated at $30^{\circ} \mathrm{C}$ for 48 hours and the antimicrobial inhibition activity for each NPs was determined by measuring the size of the clear zones surrounding the well.

Statistical analysis was accomplished using SPSS (version 16.0; SPSS Inc, Chicago, IL, USA). One-way analysis of variance (ANOVA) was applied to determine statistically significant differences among NPs fruits extracts. A post hoc analysis test LSD was functioned to examine the difference means against standard control ( $p=0.05$ or 0.01$)$. 


\section{Results and Discussion}

Screening in vitro antimicrobial activity of three stages AgNPs of Zizphus spina-christi fruits against human pathogenic microbes using agar well diffusion methods was estimated based on diameter of inhibition zone (Fig. 1). One-way ANOVA analysis clearly showed that there was significant variation in the antimicrobial killing properties of NPs depending on the fruits stages and/or the types of the tested microbial strains. No antimicrobial activity was observed against tested microbial strains using DMSO as a negative control, while the positive control (Cefoxitin$30 \mathrm{mcg}$ ) showed an inhibition zone against all tested microbes. Apparently Ag-NPs generated from Z. spina-christi ripened fruits more potent than unripened one whereas young fruits showed the lowest activity. In case of inhibition zone, the relative efficacy of NPs of Z. spina-christi from young fruits was as follows: $S$. flexneri $=P$. aeruginosam $\geq C$. albicans $\geq P$. mirabilis $\geq K$. oxytoca $\geq M$. luteus $\geq S$. aureus between $2.37 \pm 0.06$ and $1.43 \pm 0.21 \mathrm{~cm}$. Unripened NPs of $Z$. spina-christi showed moderate inhibition activity $(3.23 \pm 0.15$ to $2.65 \pm 0.09 \mathrm{~cm})$ with relative efficacy of $C$. albicans $\geq S$. flexneri $=K$. oxytoca $\geq M$. luteus $=P$. aeruginosa $\geq S$. aureus $\geq P$. mirabilis. Ripened fruits NPs showed the highest inhibition activity against all tested microbes in the range between $3.73 \pm 0.06$ and $3.40 \pm 0.26 \mathrm{~cm}$ with relative efficacy of $C$. albicans $\geq P$. aeruginosa $\geq S$. flexneri $\geq P$. mirabilis $\geq M$. luteus $\geq S$. aureus $\geq K$. oxytoca.

The obtained silver nanoparticles by the reduction of silver ions may be the result of addition fruit extract which was confirmed by UV-Vis spectroscopy. The spectrum of UV-Vis absorption of 'Green' silver nanoparticles in the existence of Z. spina-christi extract is presented in Fig. 2. Each solution color was shifted from pale yellow to the yellowish brown to more deep brown depending on the stages of fruit due to surface excitation of plasmon vibration of the silver nanoparticles. It can be seen that the surface plasmon resonance (SPR) of AgNPs was 423, 438 and $428 \mathrm{~nm}$ for ripened, unripened and young fruits, respectively (Table 1).

Table 1. Grain size (nm)) and absorption band (nm) for NPs of young, unripened and ripened fruits of $Z$. spina-christi.

\begin{tabular}{lcc}
\hline Sample & $\begin{array}{c}\text { Grain size }(\mathrm{nm}) \\
(\mathrm{XRD})\end{array}$ & $\begin{array}{c}\text { Absorption band } \\
(\mathrm{nm})\end{array}$ \\
\hline Ripened fruits & 16.50 & 423 \\
Unripened fruits & 23.25 & 438 \\
Young fruits & 50.76 & 428 \\
\hline
\end{tabular}

Fig. 3 shows the SEM images of NPs using Z. spina-christi fruits successive stages. It shows that the AgNPs for ripened and un-ripened sample were almost spherical and well dispersed while young fruits had a mixed shape. SEM image also showed more bioactive compounds as the fruits fully ripened causing AgNPs to be smaller making them more stable by preventing agglomeration.

EDX study confirmed the existence of Ag in the synthesized AgNPs. The EDX spectrum showed strong peaks of metallic silver at $3 \mathrm{kv}$, whereas the formation of silver nanoparticles has been confirmed (Fig. 3).

Fig. 4 shows the X-ray diffraction (XRD) patterns of dried silver nanoparticles synthesized using three stages of fruits ripening at room temperature. The XRD patterns of NPs indicated that the structure of silver nanoparticles is face-centered cubic, (fcc) JCPDS-4-0783. The XRD peaks at $2 \theta$ of $38.89^{\circ}, 44.31^{0}, 64.82^{\circ}$, could be attributed to the 111,200 and 220 crystallographic planes, respectively. The other observed peaks might be due to the crystallization of bio-organic phase occurring on the surface of the silver nanoparticles. It is clear from the XRD pattern that the 
AgNPs formed using fruits extract of Z. spina-christi were essentially crystalline. The average crystallite size according to Debye-Scherrer equation calculated was found to be $16.5,23.25$ and $50.76 \mathrm{~nm}$ for ripened, un-ripened and young fruits extracts, respectively.
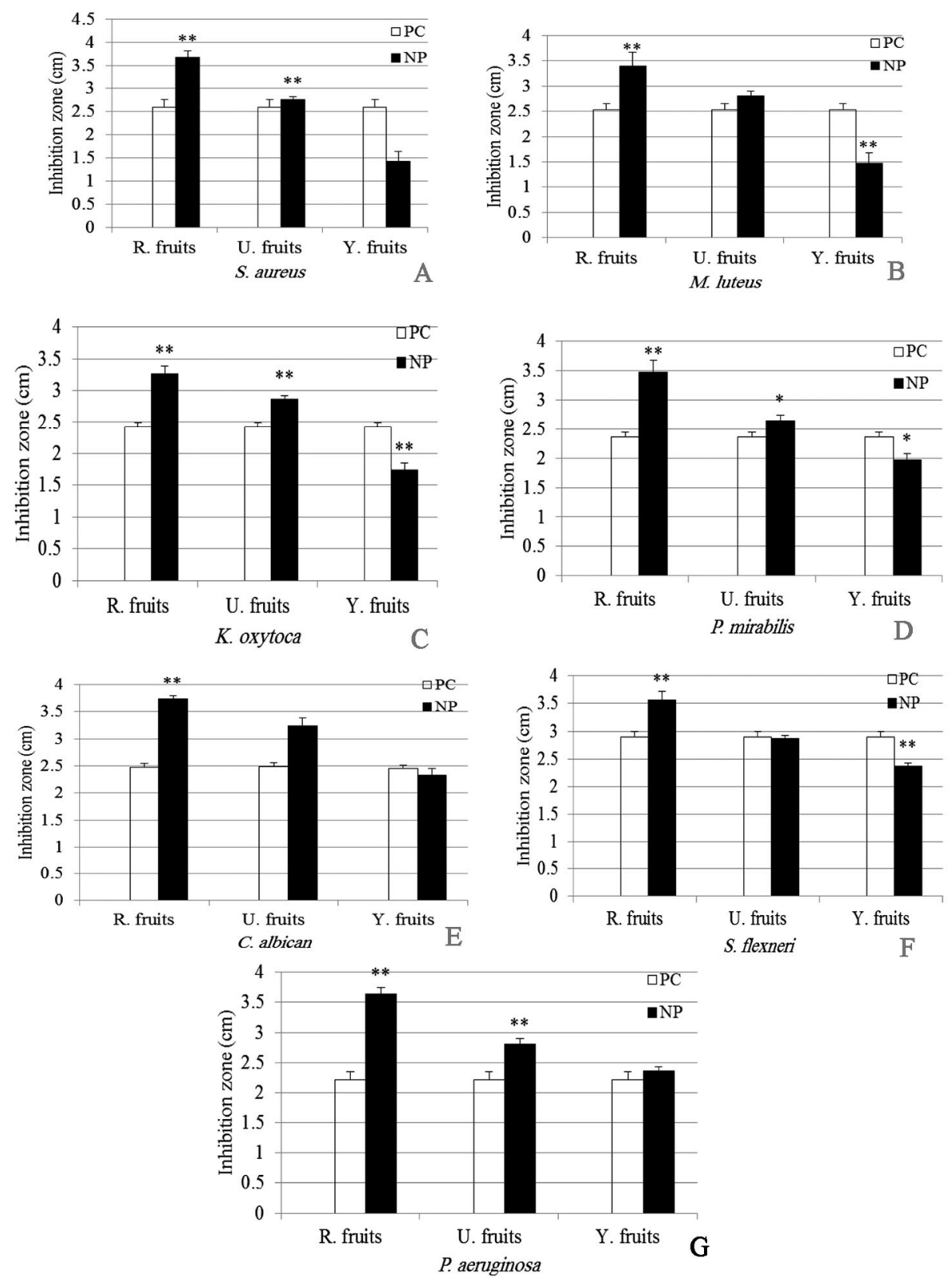

Fig. 1. Antimicrobial activity of nanoparticle extracts of Z. spina-christi against S. aureus (A), M. luteus (B), $K$. oxytoca (C), P. mirabilis (D), C. albican (E), S. flexneri (F) and P. aeruginosa (G). R. fruits (ripened fruits), U. fruits (unripened fruits, Y. fruits (young fruits). PC, positive control; significant differences $(* p \leq 0.05 ; * * \leq 0.01)$, between treatments \pm SD of the mean for $\mathrm{n}=3$. 
Biologically synthesized AgNPs have been confirmed to be used as promising medicinal molecules with substantial value as antimicrobial activities (Dicastillo et al. 2020, Tran et al. 2020). Nowadays the quest for new nanoparticles with specific biological, physical and chemical characteristics is still at the cutting edge of nanoscience research. Ziziphus species are commonly administered to treat many diseases viz., digestive complaints, causes of general weakness, hepatic disease, diabetes mellitus, as a dermatological whitening agent in skin care products and have antitumor activity for HepG2 cells (Salimi et al. 2016, Shan et al. 2019). In this research, synthesis

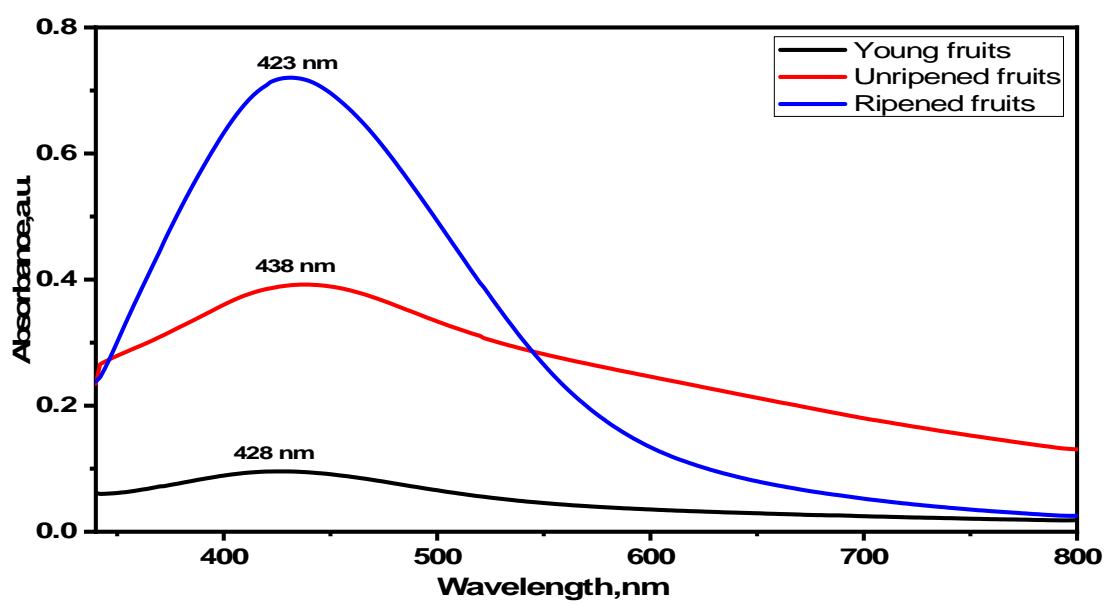

Fig. 2. UV-visible spectra of Ag-NPs solutions synthesized from different stages of fruit extract of Z. spina-christi.
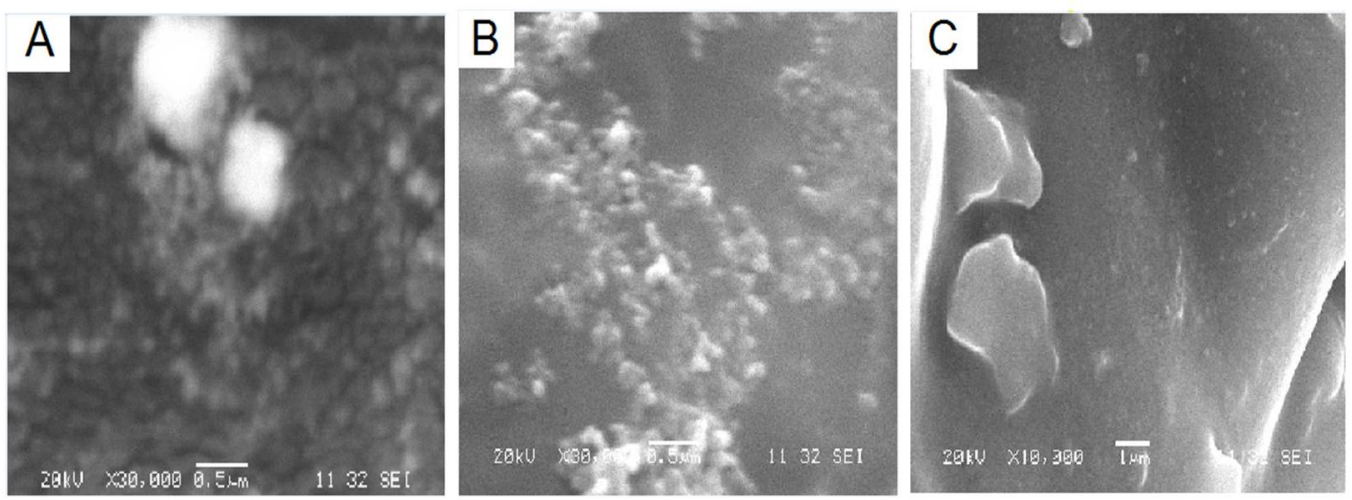

Fig. 3. SEM images of Ag-NPs from Z. spina-christi fruits. A - ripened fruit, B - unripened fruit and $\mathrm{C}$ - young fruit.

and characterization of Z. spina-christi AgNPs through various stages of fruits maturation are recorded, which may be important for pharmaceuticals aspects. AgNPs mediated by Z. spinachristi AgNPs various fruits extract documented their antimicrobial behavioral activity against human pathogens. Results showed that synthesis of AgNPs happened after subjecting silver nitrate to Z. spina-christi extract even at different stages of ripening and hence the alteration to dark brown color was remarked due to the formation of nanoparticles, which further supported by ultraviolet - visible spectroscopy (Fig. 5). Previous work documented that NPs from fruits extract 
had antimicrobial activity, for example, the biosynthesized AgNPs fruit extract of Phoenix dactylifera showed substantial antimicrobial activity against human pathogens (Zafar and Zafar 2019). There were AgNps from some fruit varieties of Carica papaya, Punica granatum, Vitis amurensis, Citrus reticulata, Ananas comosus, Vitis vinifera and Ficus carica that showed significant antimicrobial activities (Samrot et al. 2018). The present results clearly showed that NPS of Z. spina-christi relative efficacy against pathogenic microbes was as follows: ripened fruit $>$ unripened fruits > young fruits. It was well described that $95 \%$ ethanol extract of the ripened fruit of Asimina triloba exhibited effective inhibition activity against various microorganisms (Nam et al. 2019), and ripened fruit extracts from Momordica cochinchinensis showed potent antibacterial activities than the unripened fruit extracts (Tinrat 2014).
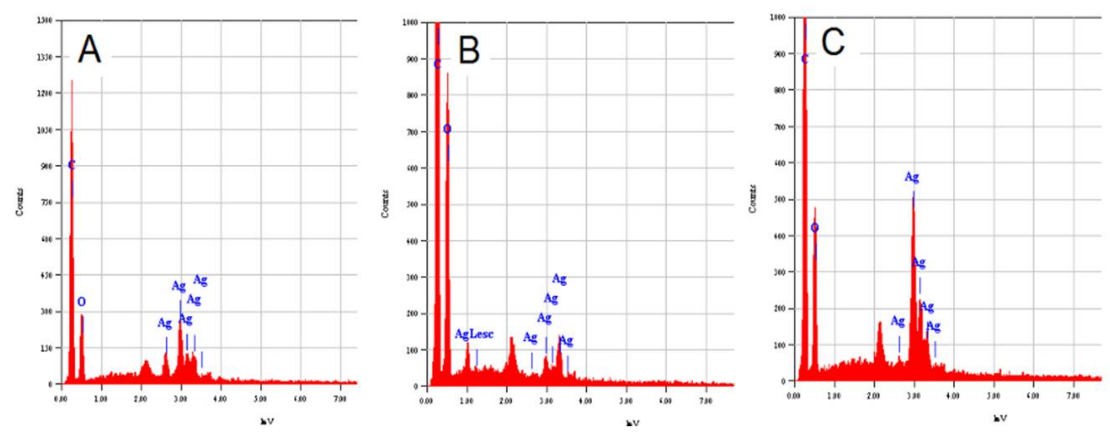

Fig. 4. EDX of Z. spina-christi NPs fruits. A. - Ripened fruit, B. - Unripened fruit, C.- Young fruit.

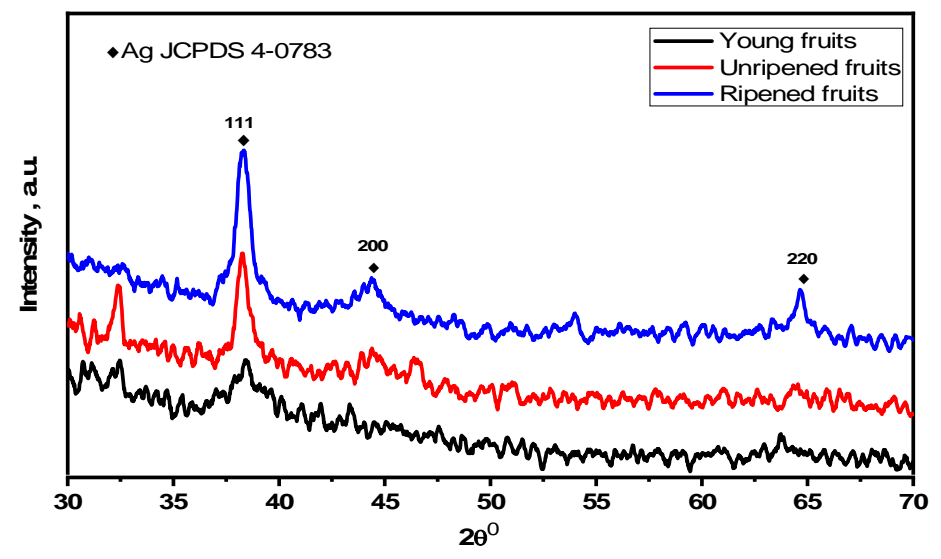

Fig. 5. X-ray diffraction (XRD) pattern of the synthesized NPs from fruits extract of Z. spina-christi.

Biosynthesized AgNPs had absorption bands for all three extracts in around $430 \mathrm{~nm}$ due to their surface plasmon resonance (SRP) attributes. Herein, the strength of the color alteration from light yellowish to dark brown probably related to the form of extract types from matured, unripened or young fruits and this is possibly due to the stimulation of the longitudinal plasmon vibrations and the reduction of $\mathrm{AgNO}_{3}$ (Kumar and Mamidyala 2011).

Specific peaks of the three-theta value in XRD pattern showed the existence of AgNPs with face-centered cubic (fcc) silver planes (Sathyavathi et al. 2010). SEM images of biosynthesized AgNPs showed that most of the particles were nearly spherical in shape and crystalline in nature with various grain sizes. Numerous sizes of spherical AgNPs with bio-organic compound 
crystallization had been documented using TEM and SEM studies (Elbeshehy et al. 2015). It has been stated that the configuration of nanoparticles significantly affects the optical and electronic property of metallic nanoparticles (Sankar et al. 2013).

EDX signals confirmed the presence of a silver element in the synthesized AgNPs with a peak optical absorption range, which was observed in the biosynthesized AgNPs using the three stages of fruit ripening processes. Metallic silver nanocrystals typically displayed standard EDX signals, widely known for the absorption of metallic silver (Jyoti et al. 2016). In conclusion, this research offers an economical, environment-friendly and reproducible approach to the synthesis of AgNPs using $Z$. spina-christi fruit extracts at various stages of maturation as a reducing, stabilizing and capping agent. This research also demonstrated that the growth of human pathogens could be inhibited by the synthesized AgNPs fruit extract of various stages. Therefore, it could be used as potential antimicrobial agents.

\section{Acknowledgements}

The authors extend their appreciation to the Dean of Scientific Research at King Khalid University for funding under grant number (R.G.P2/90/41).

\section{References}

Amin A and Mahmoud-Ghoneim D 2009. Zizyphus spina-christi protects against carbon tetrachlorideinduced liver fibrosis in rats. Food Chem. Toxicol. 47: 2111-2119.

Bianco C, Visser MJ, Pluut OA, Svetličić V, Pletikapić G, Jakasa I, Riethmuller C, Adami G, Larese Filon F and Schwegler-Berry D 2016. Characterization of silver particles in the stratum corneum of healthy subjects and atopic dermatitis patients dermally exposed to a silver-containing garment. Nanotoxicology 10: $1480-1491$.

Collenette S 1998. Wild Flora of Saudi Arabia. International Asclepiad Society Publications Ltd., London.

Dicastillo CLd, Vidal CP, Falcó I, Sánchez G, Márquez P and Escrig J 2020. Antimicrobial bilayer nanocomposites based on the incorporation of As-Synthetized Hollow Zinc Oxide Nanotubes. Nanomaterials 10: 503.

Duke JA and Ayensu ES 1985. Medicinal plants of China, Reference Publications.

El-Kamali H and El-Khalifa K 1999. Folk medicinal plants of riverside forests of the southern blue nile district, Sudan. Fitoterapia 70: 493-497.

Elbeshehy EK, Elazzazy AM and Aggelis G 2015. Silver nanoparticles synthesis mediated by new isolates of Bacillus spp., nanoparticle characterization and their activity against bean yellow mosaic virus and human pathogens. Front. Microbiol. 6: 453.

Jyoti K, Baunthiyal M and Singh A 2016. Characterization of silver nanoparticles synthesized using Urtica dioica Linn. leaves and their synergistic effects with antibiotics. J. Radiat. Res. Appl. Sci., 9: 217-227.

Kadioglu O, Jacob S, Bohnert S, Naß J, Saeed ME, Khalid H, Merfort I, Thines E, Pommerening T and Efferth T 2016. Evaluating ancient Egyptian prescriptions today: anti-inflammatory activity of Ziziphus spina-christi. Phytomedicine 23: 293-306.

Kumar CG and Mamidyala SK 2011. Extracellular synthesis of silver nanoparticles using culture supernatant of Pseudomonas aeruginosa. Colloids Surf. B. Biointerfaces 84: 462-466.

Mandaville JP and Mandaville JP 1990. Flora of Eastern Saudi Arabia, Kegan Paul International London.

Moustafa MF and Alrumman SA 2015. First report about pharmaceutical properties and phytochemicals analysis of Rosa abyssinica R. Br. ex Lindl.(Rosaceae). Pak. J. Pharm. Sci. 28(6): 2009-17.

Moustafa MF, Hesham AE-L, Quraishi MS and Alrumman SA 2016. Variations in genetic and chemical constituents of Ziziphus spina-christi L. populations grown at various altitudinal zonation up to $2227 \mathrm{~m}$ height. J. Genet. Eng. Biotechnol. 14: 349-362. 
Nam JS, Park SY, Oh HJ, Jang HL and Rhee YH 2019. Phenolic profiles, antioxidant and antimicrobial activities of Pawpaw Pulp (Asimina triloba [L.] Dunal) at different ripening stages. J. Food Sci. 84: 174182.

Saied AS, Gebauer J, Hammer K and Buerkert A 2008. Ziziphus spina-christi (L.) Willd.: a multipurpose fruit tree. Genet. Resour. Crop Evol. 55: 929-937.

Salimi M, Sarkhail P, Sarkheil P, Mostafapour Kandelous H and Baeeri M 2016. Evaluation of antimelanogenic activity of Ziziphus jujuba fruits obtained by two different extraction methods. Res. J. Pharmacogn., 3: 1-7.

Samrot AV, Raji P, Selvarani AJ and Nishanthini P 2018. Antibacterial activity of some edible fruits and its green synthesized silver nanoparticles against uropathogen - Pseudomonas aeruginosa SU 18. Biocatal Agric Biotechnol. 16: 253-270.

Sankar R, Karthik A, Prabu A, Karthik S, Shivashangari KS and Ravikumar V 2013. Origanum vulgare mediated biosynthesis of silver nanoparticles for its antibacterial and anticancer activity. Colloids Surf. B. Biointerfaces 108: 80-84.

Sathyavathi R, Krishna MB, Rao SV, Saritha R and Rao DN 2010. Biosynthesis of silver nanoparticles using Coriandrum sativum leaf extract and their application in nonlinear optics. Adv. Sci. Lett. 3: 138-143.

Shan S, Xie Y, Zhao H, Niu J, Zhang S, Zhang X and Li Z 2019. Bound polyphenol extracted from jujube pulp triggers mitochondria-mediated apoptosis and cell cycle arrest of HepG2 cell in vitro and in vivo. J. Funct. Foods 53: 187-196.

Tinrat S 2014. Comparison of antioxidant and antimicrobial activities of unripe and ripe fruit extracts of Momordica cochinchinensis Spreng (Gac fruit). Int. J. Pharm. Sci. Rev. Res. 14: 75-82.

Townsend C and Guest E 1974. Flora of IRAQ. Ministry of Agriculture and Agrarian rEform, Iraq.

Tran DN, Vu NN, Nhan T, Bich NTT, Quang ML, To NB, Le Van P and Dang VQ 2020. Silver nanoparticles as potential antiviral agents against African swine fever virus. Mater. Res. Express., 6: 12.

Zafar S and Zafar A 2019. Biosynthesis and characterization of silver nanoparticles using phoenix dactylifera fruits extract and their in vitro antimicrobial and cytotoxic effects. Open Biotechnol J. 13: 37-46.

(Manuscript received on 28 May, 2019; revised on 5 August, 2020) 